\title{
DEM Calibration Concept for TanDEM-X
}

\author{
J. Hueso González, M. Bachmann, H. Fiedler, S. Huber, G. Krieger, M. Zink \\ Microwaves and Radar Institute, Oberpfaffenhofen \\ German Aerospace Center (DLR) \\ D-82234 Wessling, Germany \\ jaime.hueso@dlr.de
}

\begin{abstract}
The TanDEM-X mission [1] comprises two fully active synthetic aperture radar satellites operating in $X$-band. The primary goal of this mission is the derivation of a high-precision global Digital Elevation Model (DEM) according to HRTI level 3 quality [2]. This requires accurate calibration of the interferometric system parameters. Content of this paper is the development of a general concept for this calibration, which comprises the determination of instrument and baseline errors, an adjustment concept and the distribution of control points. This concept has a key incidence on mission aspects like the data acquisition plan and the data take adjustment procedure.
\end{abstract}

Keywords: calibration, radar interferometry, synthetic aperture radar, phase noise.

\section{INTRODUCTION AND OBJECTIVES}

The challenge of calibrating the TanDEM-X DEM lies on the complexity of the system and the strong height requirements. The interferometric height, which the DEM is based on, is determined by the phase difference between the two bi-static images and the spatial geometry. Additionally, baseline errors intrinsic of the bi-static SAR configuration combined with errors and drifts of the radar instrument add to the real interferometric height and lead to a faulty height information. Thus, the DEM has to be corrected to achieve the accuracies defined (Table 1). The adjustment techniques take into consideration the nature of the errors and allow generating an adjusted scenario with the information coming from the set of data takes available in the interest region.

TABLE I. TANDEM-X DEM SPECIFICATIONS

\begin{tabular}{|l|l|l|}
\hline \multicolumn{1}{|c|}{ Requirement } & \multicolumn{1}{|c|}{ Specification } & \multicolumn{1}{c|}{ HRTI-3 } \\
\hline $\begin{array}{l}\text { Absolute vertical accuracy } \\
(\text { global })\end{array}$ & $90 \%$ linear error & $10 \mathrm{~m}$ \\
\hline $\begin{array}{l}\text { Relative vertical accuracy } \\
(100 \mathrm{~km} \times 100 \mathrm{~km})\end{array}$ & $\begin{array}{l}90 \% \text { linear point-to- } \\
\text { point error }\end{array}$ & $\begin{array}{l}2 \mathrm{~m}(\text { slope }<20 \%) \\
4 \mathrm{~m}(\text { slope }>20 \%)\end{array}$ \\
\hline
\end{tabular}

The calibration concept presented here, developed for the TanDEM-X Preliminary Design Review, provides essential data to extend the strategy for the data acquisition plan [3] in order to obtain global coverage within the mission time. In addition, it offers enough information to allow the application of bundle block adjustments [4] to the raw DEMs.

\section{ACQUISITION SCENARIO}

The mission scenario for TanDEM-X is designed to obtain a global coverage of the earth within mission time, and to guarantee a DEM with the specified accuracies.

All land surfaces will be covered at least twice with different heights of ambiguity $\left(\mathrm{h}_{\mathrm{amb}}\right)$ in order to support multibaseline phase unwrapping (PU).

Within the first year, the land masses will be completely covered using four different helixes and a small baseline (closer formation). The helixes will have $h_{a m b}$ between $35 \mathrm{~m}$ and $43 \mathrm{~m}$ for latitudes between -60 and $+70 \mathrm{deg}$.

The northern hemisphere will be mapped with ascending orbits, whereas the southern hemisphere with descending orbits. The length of the data takes will be maximized within the resource limits in order to simplify the adjustment.

During the second year, another global acquisition over land areas will be performed with scaled (larger) helixes delivering (predicted) relative accuracy between 1.5 and $2 \mathrm{~m}$.

Finally, about half a year will be needed to acquire crossing orbits (useful for DEM adjustment) and to cover difficult terrain $(<20 \%$ of the total land mass $)$ for the $3^{\text {rd }}$ and $4^{\text {th }}$ time under different geometry (look direction and/or incidence angles).

Another important parameter for calibration and DEM adjustment is the length of the data takes. The longer they are the easier and more accurate is the adjustment. This is so because the drifts of the satellites remain relatively constant or linear within the same acquisition period. Individual data take lengths and the mapping per orbit will be maximized within the available resource limits. The main limiting aspects are expected to be the ground station contact time, the solid state mass memory and the energy during summer eclipse periods.

As stated here, the first challenge to face is the adjustment of scenarios with all parallel orbits. However, at the end of the mission phase, the possibility of acquiring spare descending orbits in the northern and spare ascending orbits in the southern hemisphere (crossing orbits) is considered. This would help the adjustment, as will be shown in the simulations later on. The required amount of crossing swaths or ground control points will be studied.

All these fundaments give a good overview of the data take scenario the DEM calibration will face. 


\section{ERROR SOURCES}

The main sources of the arising phase errors as mentioned before can be classified in three groups: inaccuracies in the baseline determination, phase errors in the radar instruments and performance degradation due to both noise equivalent $\sigma_{0}$ (NESZ) and signal to noise ratio (SNR). Noise-like, randomly distributed contributions of these errors already exhaust most of the $2 \mathrm{~m}$ relative error margin allowed for an area of $100 \mathrm{~km} \times 100 \mathrm{~km}$. In order to keep the total error under this requirement, a relative error of $0.5 \mathrm{~m}$ has been defined as the threshold for the "low frequency" height errors.

Hence, to achieve the required accuracy, "low frequency" parts of the errors which appear as biases or drifts in terms of the length of one data take have to be minimised. Examples of systematic slow changing errors for baseline determination are inaccuracies in the relative orbit and attitude determination of the TanDEM-X helix formation and variations in the SAR antenna phase centre. On instrument side, slow errors occur due to remaining interpolation errors after internal calibration and phase drifts during synchronisation pulse sequences in the amplifiers not compensated by the internal calibration. When a data take is acquired, these phase errors lead to a height error in the resulting raw DEM.

\section{CALibration ApProACH}

The phase errors introduced in chapter 3 are translated into height errors in the raw DEM that trespass the specified height error threshold of the mission. Therefore, several data take adjustments and calibration strategies were considered.

Relative corrections can be derived by exploiting the height differences and using concurring swath overlaps and crossing orbits in the data take scenario. Especially chosen bundle block adjustment techniques are applied in different ways depending on the scenario configuration to balance the height error realisations.

Absolute height calibration requires accurate height references. The references have to be adequately distributed depending on the data take adjustment scenario. Coverage on all significant isolated land masses and a known accuracy which fulfils the requirements are pursued, with the aim of guaranteeing the correct adjustment of the elevation models by the Mosaicing and Calibration Processor (MCP).

This can be achieved by using global data sets (like ICESat height information), which provide very useful information even in regions of the planet where the access to height data is limited or unreliable. However, local height calibration targets are still necessary, due to their high accuracy, particularly in regions where global data may have blind spots and for validation purposes. As a secondary mission goal of TanDEM$\mathrm{X}$, in certain interest regions the DEM accuracy specifications will be improved to fulfil a HRTI-4 standard. In these cases, local very accurate calibration targets may play an important role.
Reference information in open terrain is preferable, because uncertainties between terrain and surface models do not need to be considered.

The simulations shown in the next chapter will assist in the definition of the requirements for the distribution, location and amount of the reference data points in a scene.

Some of the studied absolute calibration sources are listed here:

- Space borne laser radar altimeters like 'ICESat' elevation data.

- GPS/GALILEO tracks.

- Land - Sea transitions in case of good correlation.

- Local highly accurate DEMs from airborne LIDAR, Photogrammetry and SAR.

- Corner reflectors and transponders.

\section{ERror SimUlation AND AdJUSTMENT TOOLS}

Combining the above mentioned inputs, a height error simulation tool has been developed. With its help it is possible to analyse the influence of the different error sources on the system performance and to propose inputs to the acquisition scenario. The simulator includes an error generator and an adjustment module, which applies the bundle block techniques selected by the user to the calculated height error realisation. They will give an idea of the number, quality and distribution of calibration references and of crossing orbits required to successfully calibrate the global DEM. In the following simulations, the initial phase error due to $2 \pi$ phase wrapping is not included.

First, a scenario containing only parallel orbits will be adjusted. As described in chapter 2, the acquisition plan proposes to obtain two acquisitions for each orbit, which would offer an adjusting scenario similar to the one presented in Figure 1.

The parallel scenario has three major advantages:

- The same strips are mapped during the same period of time in the year. Hence, vegetation, tree cover or ice cover are seen in a similar state.

- No major formation change will be necessary during the first two years of acquisition. First after these two years, the satellite formation is changed anyway to acquire difficult terrain like mountainous regions.

- Multi baseline processing is simplified significantly.

Simulations show that three control points will be required in each of the two data takes in the borders of the scene, so they can be completely adjusted. The central data takes will be corrected by propagating the information of the external ones fixed by control points. All the control points have an accuracy of $10 \mathrm{~cm}$ (one sigma). 
One calculated error realisation is shown in Figure 2, together with the results of the adjustment (Fig. 3).

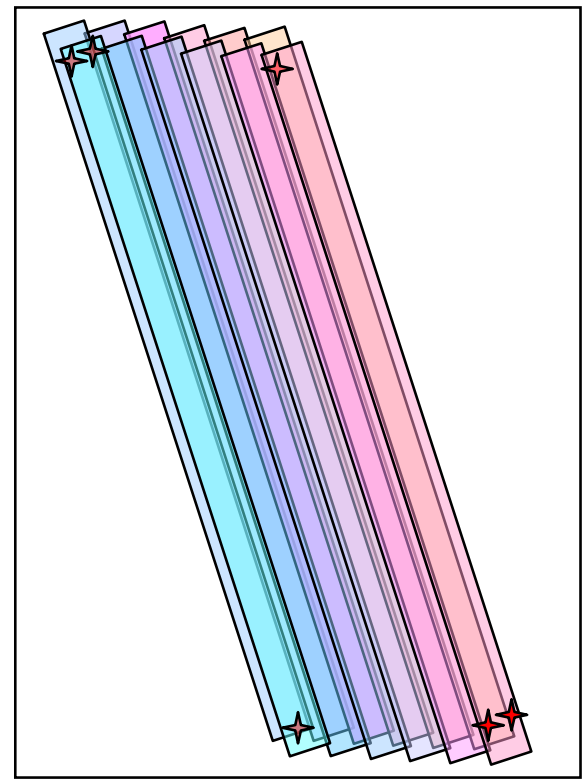

Figure 1. Double adjacent orbits.

The maximum relative error has been improved from $6.6 \mathrm{~m}$ to $0.26 \mathrm{~m}$, which is a very good correction and keeps the height error within the requirements.

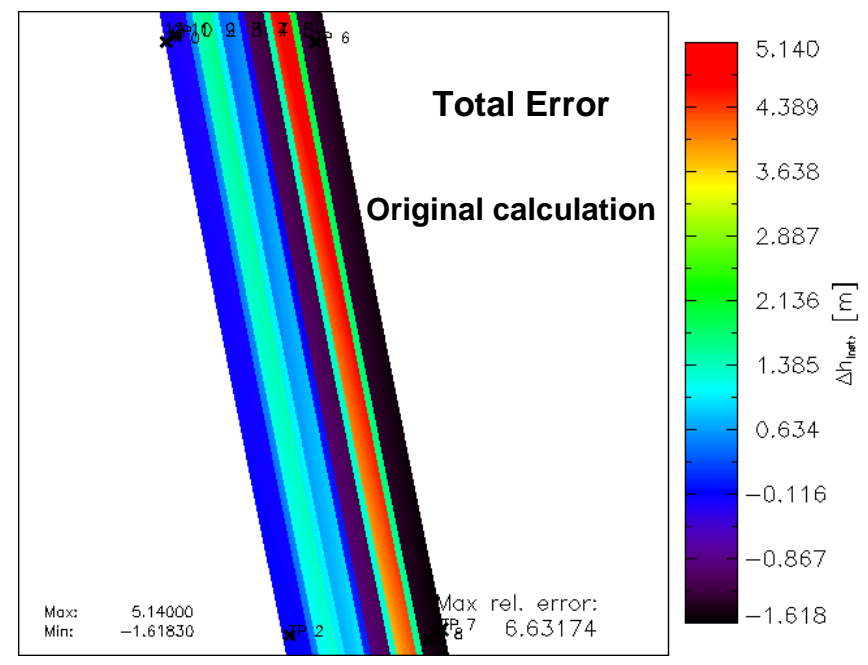

Figure 2. Error realisation and adjustment.

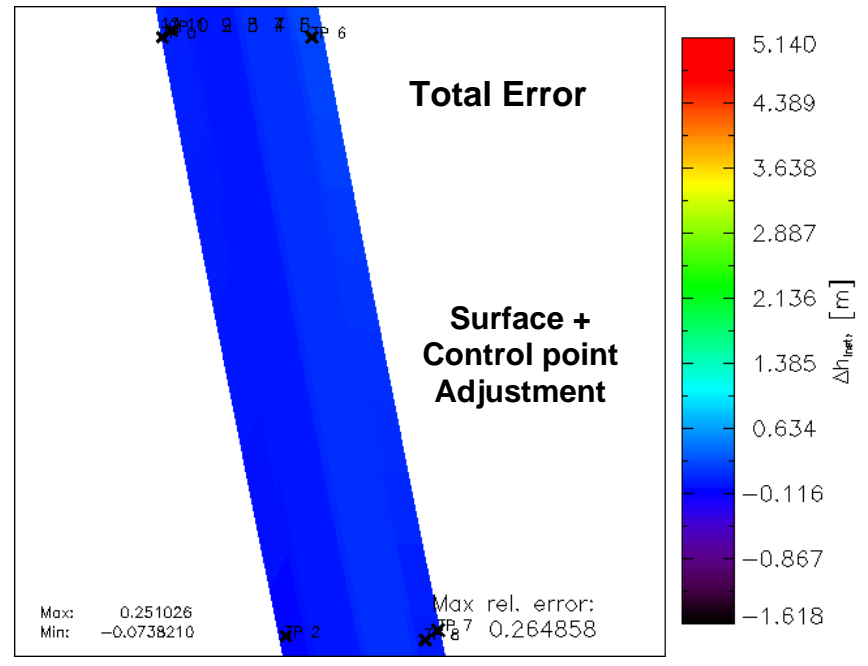

Figure 3. Error realisation and adjustment.

The advantage of crossing orbits is that they allow correcting the elevation tilts of the other realisations with less control points as in the last example and more flexibility in their location.

Two control points are needed in the crossing orbits, one on each extreme, as depicted in Figure 4. This compensates the along track drift of the crossing orbit, which will be the reference for the others. Another important conclusion of this simulation is the required distance between the crossing orbits in order to get a good error correction.

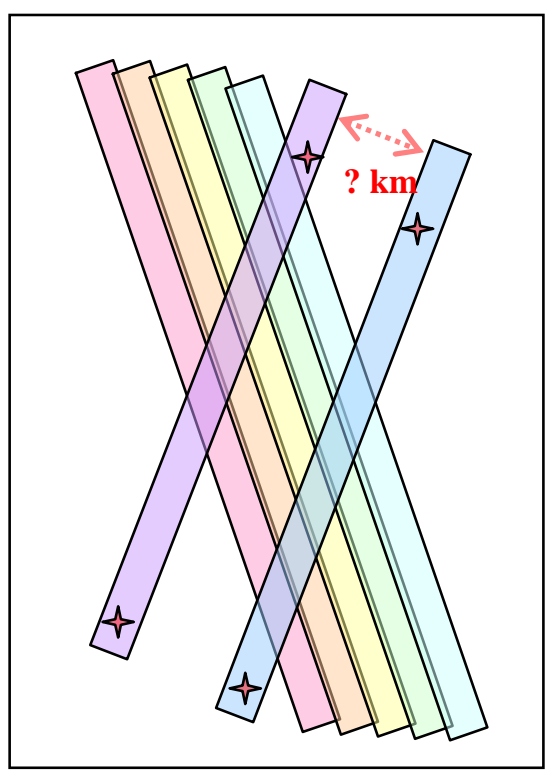

Figure 4. Ascending orbits scene with two crossing orbits

Fig. 5 shows the original height errors of the scene and Fig. 6 presents the remaining errors after applying the "tilt" method and the control point adjustment. 


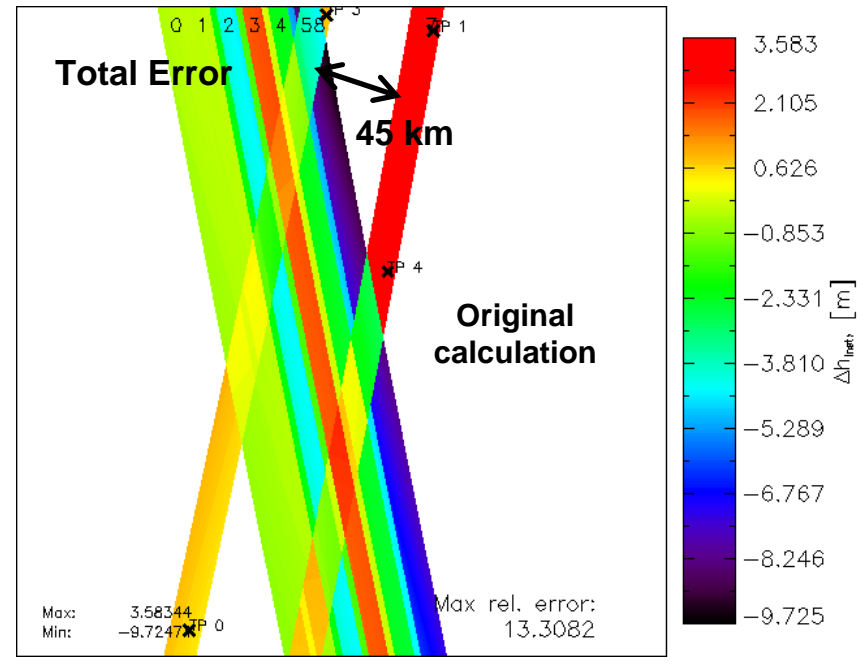

Figure 5. Error realisation and adjustment.

The maximum relative error has improved from $13 \mathrm{~m}$ to $0.45 \mathrm{~m}$, which meets our requirements $(0.5 \mathrm{~m})$.

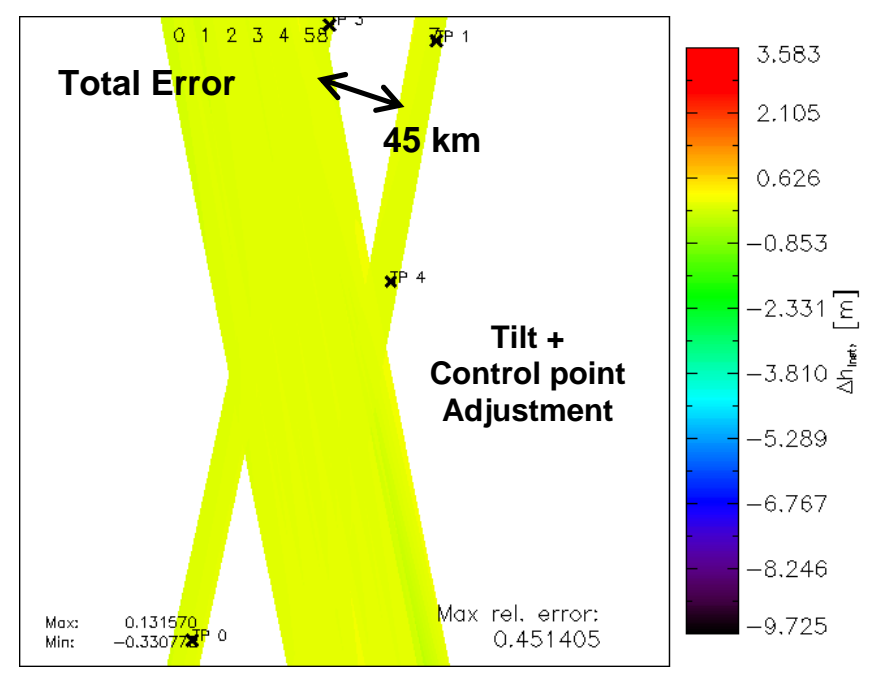

Figure 6. Error realisation and adjustment.

Some refinements in the adjustment scene are still possible. A better correction of the tilt could be achieved with an extra control point in the crossing data takes. The residual along track slope could be levelled with a bigger separation between the two crossing data takes.

The optimal separation between crossing orbits depends on the size of the adjacent scenario to adjust and on the angle between ascending and descending orbits, which varies with the latitude of the acquisition. Assuming our simulation parameters, the best separation between the crossing orbits lies around 50-60 km. The computation capability of the adjustment software limits the surface of the scenario to adjust. Improvements on the software are being performed and the minimum separation between crossing orbits will be increased, as will be proved by future simulations.

The distance of crossing orbits is un-critical as long as the instrument behaves as expected. However, it has to be big enough in order to create a stable adjustment net between the connected data takes.

\section{SUMMARY AND CONCLUSIONS}

As a preliminary step, all the phase errors caused by baseline uncertainties and instrument drifts have been carefully analysed and implemented in a new software tool.

Some "key" scenarios have been simulated and adjusted in order to validate the bundle block adjustment techniques and assess the viability of the mission scenarios and the achievement of the desired height accuracies. This has been useful to provide important inputs to the mission and acquisition plan.

During 2007, after the launch of the first satellite of the tandem, TerraSAR-X, its calibration and validation results will offer essential information about the satellite operation, which will allow refining the performance prediction of the whole TanDEM-X system. These data will also be used to validate and improve the calibration concept presented in this paper.

\section{ACKNOWLEDGMENT}

The TanDEM-X project is partly funded by the German Federal Ministry for Economics and Technology (Förderkennzeichen 50 EE 0601).

\section{REFERENCES}

[1] A. Moreira et al. "TanDEM-X: A TerraSAR-X Add-On Satellite for Single-Pass SAR Interferometry", in Proc. IGARSS, Anchorage, USA, 2004.

[2] “HRTI-3-DEM draft document”, NGA, MIL PRF 89048.

[3] H. Fiedler, G. Krieger, M. Werner, K. Reiniger, M. Eineder, S. D'Amico, R. Klein, D. Erhardt, M. Wickler. "The TanDEM-X Mission Design and Data Acquisition Plan", EUSAR, Dresden, Germany, 2006.

[4] B. Triggs, P. McLauchlan, R. Hartley, and A. Fitzgibbon. "Bundle adjustment - a modern synthesis. Vision Algorithms: Theory \& Practice”. Springer-Verlag, 2000. 